Mondes du Tourisme

du tourisme

$2 \mid 2010$

Vers une théorisation de l'approche géographique du tourisme

\title{
Pratiques touristiques de et en diaspora
}

Quand les Indiens prennent possession du monde

\section{Anthony Goreau-Ponceaud}

\section{(2) OpenEdition \\ Journals}

Édition électronique

URL : http://journals.openedition.org/tourisme/278

DOI : $10.4000 /$ tourisme. 278

ISSN : 2492-7503

Éditeur

Éditions touristiques européennes

Édition imprimée

Date de publication : 1 décembre 2010

Pagination : 70-86

ISSN : 2109-5671

Référence électronique

Anthony Goreau-Ponceaud, "Pratiques touristiques de et en diaspora », Mondes du Tourisme [En ligne], 2 | 2010, mis en ligne le 30 septembre 2015, consulté le 19 avril 2019. URL : http://

journals.openedition.org/tourisme/278; DOI : 10.4000/tourisme.278

Licence Creative Commons

Mondes du tourisme est mis à disposition selon les termes de la licence Creative Commons Attribution

- Pas d'Utilisation Commerciale - Pas de Modification 4.0 International. 


\title{
Pratiques touristiques de et en diaspora
}

\section{Quand les Indiens prennent possession du monde}

\author{
ANTHONY GOREAU-PONCEAUD \\ Maître de conférences, université de Bordeaux 4, UMR 5185 ADES CNRS
} [anthonygoreau@yahoo.fr]

Résumé. En s'appuyant sur deux séries d'enquêtes, l'une menée durant mon doctorat de géographie sur la diaspora tamoule, l'autre en cours depuis avril 2010, cet article propose d'explorer les relations entre tourisme et diaspora, d'une part, et de déterminer de quelle manière le tourisme d'une diaspora au sein d'un espace diasporique (de et en diaspora) est une condition de réalisation de l'expérience diasporique, d'autre part. Dans un premier temps, cela implique de définir l'espace diasporique, mais aussi de préciser que celui-ci n'est pas un lieu autre - possédant un degré d'altérité - pour ses membres, mais plutôt une forme d'hétérotopie. Dans un second temps, je pose l'hypothèse que le tourisme diasporique est une condition nécessaire, pour ces transmigrants, à la réalisation de l'expérience diasporique. En d'autres termes, le tourisme est diasporique parce qu'il participe de la diaspora, mais également parce qu'il contribue à organiser l'espace diasporique d'une nouvelle manière, déterminant un savoir être d'ici et d'ailleurs.
Abstract. Based on surveys conducted during my Ph.D. in geography on the Tamil diaspora, this article proposes, first, to explore the relationship between tourism and diaspora, and, secondly, to determine how diasporic tourism is a condition for the realization of the diasporic experience. This implies in a first move to define the space of diaspora, i.e. not as an "other" place with a certain degree of otherness, but rather as a form of heterotopia. In a second move, I assume that diasporic tourism is a necessary condition, for these "transmigrants", to achieve their diasporic experience. In other words, tourism is diasporic because it participates in the diaspora, and because it helps to organize the diasporic space in a new way. Thus it develops people's know-how to belong to a "here" and to be from "elsewhere". 
$\mathbf{A}$ u cours de mon doctorat de géographie, j’ai enquêté pendant quatre ans sur les trajectoires spatio-temporelles et les inscriptions territoriales de la diaspora issue du monde indien, et plus particulièrement tamoule ${ }^{(1)}$, en Île-de-France. En mobilisant les outils de "l'ethnologie multisite", j’ai cherché à comprendre ce qui pouvait créer de l'unité dans l'émiettement (George, 1998). J'ai donc mené, entre 2004 et 2008, une enquête sur plusieurs sites, à la fois en Île-de-France et en Inde, mêlant questionnaires et entretiens, sur un échantillon total de 523 personnes (284 enquêtes en Île-de-France et 239 en Inde, menées en langue française et anglaise $)^{(2)}$. Après une analyse de ces résultats, il m'est apparu comme une évidence que "l'existence de fondements à la présence sur un lieu [permet] d'ouvrir de nouveaux lieux dans des non-lieux" (Hovanessian, 2007, p. 12). J'ai alors focalisé mon analyse sur les récits d'une nouvelle territorialisation, sur la refondation d'un langage collectif et de nouveaux cadres sociaux de la mémoire et sur les dynamiques de transmission entre générations, à partir des fragments réappropriés d'une histoire souvent malmenée (Halbwachs, 1994).

Cependant, cela restait insuffisant et ne répondait que partiellement à une question essentielle : comment tenir malgré la distance? Plusieurs récits de vie et questionnaires (52 au total $)^{(3)}$ m'ont permis de dégager de nouveaux éléments de réponse : la construction et l'organisation de réseaux. Dans un monde globalisé, au sein duquel la circulation, les migrations et le mouvement bouleversent la formation des identités personnelles et collectives, la question de l'habiter et du chez-soi, sousjacente à l'organisation en réseau, prend une importance significative dans le cas des communautés diasporiques. En effet, de plus en plus de communautés diasporiques se rendent dans leur pays d'origine dans un but identitaire, afin de "re-root their identities and find nourishment" [enraciner de nouveau leurs identités et les nourrir] (Basu, 2004, p. 28).

Je pose comme hypothèse que le tourisme pourrait alors être analysé comme un moyen par lequel les relations transnationales entre les différents points d'ancrage de la diaspora indienne sont maintenues et renforcées. En cela, le tourisme de et en diaspora serait un acte par lequel les membres de la diaspora indienne se constituent socialement. Il aurait donc des effets performatifs dans la formation de territoires mobiles dont les contours font certes appel à la mémoire, mais également à des attachements multiples. Ces pratiques permettraient de faire la conquête de l'espace diasporique, car elles constitueraient un puissant opérateur de mise en circulation des individus au sein de ce même espace. Elles ne seraient donc pas un facteur de déstructuration, voire de destruction (des identités), mais au contraire un élément favorisant la co-construction d'une identité transnationale, hybride, en somme diasporique. Plusieurs chercheurs, parmi lesquels Paul Basu, ont tenté de décrire ces pratiques de retour en tant que "roots tourism" (Basu, 2004). Je n'utiliserai pas cette terminologie, lui préférant celle de tourisme de diaspora, suggérant dans les lignes qui suivent que la diaspora, en tant que rapport à l'espace, serait l'archétype d'une nouvelle façon d'habiter le monde (Stock, 2006).

Par ailleurs, quels sont les effets des phénomènes de diaspora sur les formes du tourisme contemporain ? De quelles manières participent-ils à la mise en tourisme des lieux ? Quelles sont les façons de pratiquer touristiquement un espace diasporique ? Quels sont les opérateurs sociaux de cette forme particulière de tourisme ? Quelle part prend le "référent origine" (c'est-à-dire le pays d'origine, de référence) dans l'organisation et/ou l'institutionnalisation de ces pratiques ? Enfin, le "diasporé" est-il réellement un touriste ? ou est-ce un résident ou encore un "revenant épisodique" (Urbain, 2002) ?

Répondre à ce faisceau d'interrogations implique, dans un premier temps, de définir la diaspora en tant qu'espace, mais également de préciser que celle-ci peut influencer la forme et les évolutions 
contemporaines des pratiques touristiques en Inde, et dans les autres pôles où sont établis des Indiens. Dans un deuxième temps, il s'agira de comprendre, à partir d'un poste d'observation privilégié, Pondichéry, ce qui est en jeu par ce type de déplacement touristique. Nous insisterons alors sur l'émergence de nouvelles pratiques touristiques et sur la façon dont cellesci s'inscrivent dans les flux globaux (ceux de la diaspora indienne) nous obligeant à repenser les catégories Nord et Sud comme des modes de lecture pertinents dans l'analyse du tourisme.

\section{LA DIASPORA COMME RAPPORT SPÉCIFIQUE À L'ESPACE}

Le mot "diaspora” a connu ces trois dernières décennies une inflation d'usages pour désigner aujourd'hui des processus migratoires très divers. L'extraordinaire plasticité sémantique du terme ouvre des horizons de pensée, de débat et de réflexion très larges - y compris dans le champ des études touristiques. Le terme s'accompagne de deux dimensions heuristiques: la première tient en sa capacité à interpeller toutes les disciplines des sciences sociales; la seconde à rendre concrètes et particulièrement pertinentes les interrogations qui portent sur les relations dialectiques

Tableau 1

Critères communs d'une diaspora selon Cohen (Cohen, 1997, p. 26)

\begin{tabular}{ll}
\hline I & Dispersion depuis une patrie d'origine, souvent de manière traumatique, vers au moins deux pays étrangers. \\
\hline 3 & L'expansion depuis une patrie pour une recherche de travail, des activités commerciales, ou des ambitions coloniales. \\
\hline 4 & Une mémoire collective et un mythe à propos de la patrie d'origine. \\
\hline 5 & Une idéalisation de l'origine ancestrale supposée. \\
\hline 6 & Un mouvement de retour. \\
\hline 7 & Une relation ambiguë ou difficile avec les sociétés d'accueil. \\
\hline 8 & Un sens de la solidarité vis-à-vis des membres du groupe dans d'autres pays. \\
\hline 9 & La possibilité d'une vie distinctive, créative, et enrichissante au sein des sociétés tolérantes et pluralistes.
\end{tabular}

Tableau 2

Types de diaspora selon Cohen (Cohen, 1997, p. 178)

\begin{tabular}{|c|c|}
\hline Terminologie jardinière & Type de diaspora \\
\hline Désherber (I) & Victime, réfugiée \\
\hline Ensemencement (2) & Impériale, coloniale \\
\hline Repiquer, transplanter (3) & Travail, service \\
\hline Marcotter (4) & Commerce, affaires \\
\hline Pollinisation (5) & Culturelle, hybride, postmoderne \\
\hline
\end{tabular}

\section{Exemples}

Juive, africaine, arménienne

Russe, britannique, grecque

Indienne, chinoise, japonaise ; sikhe, turque, italienne

Libanaise, chinoise, indienne

Caribéenne, chinoise, indienne

( I) Expulsion, déportation, génocide. (2) Dissémination, correspond à l'origine étymologique grecque du verbe speirein [semer]. (3) Déterrer et replanter. C'est le cas en particulier des coolies indiens et chinois. Une grande part de l'échec de la transplantation est liée aux conditions de départ, du voyage et du site d'arrivée. (4) Procédé de multiplication végétative des plantes, par lequel une tige aérienne est mise en contact avec le sol et s'y enracine avant d'être isolée de la plante mère. (5) Dans le cas des diasporas culturelles, la pollinisation est assurée par de nouvelles arrivées (mouvements migratoires) et par les ondes (migrations des idées et de la musique par exemple). 
qui se nouent entre l'individu et le social, entre l'hétérogénéité et l'homogénéité, entre le territoire et le réseau. Ces dimensions heuristiques visent à susciter un travail épistémologique et méthodologique pour rendre explicites les tenants et les aboutissants du processus de catégorisation. Car quels éléments différencient aujourd'hui un groupe de migrants d'une diaspora ? Dès lors, si différence il y a, comment ce processus s'effectue-t-il ? Quels en sont les structures, les composantes, les modalités et les champs d'action ? Et que voulons-nous signifier et caractériser en utilisant la catégorie diaspora?

Voilà un ensemble de questions qui interrogent, à juste titre, la capacité des mots à correspondre encore à des réalités marquées par des changements significatifs. Face aux ambiguïtés qui se cachent derrière l'apparent pouvoir rassembleur du terme diaspora, la mobilité intrinsèque liée à ce phénomène, d'essence à la fois spatiale et sociale, soulève fondamentalement la question de l'habiter et de l'identité ; elle pose comme problématique la complexité de l'existence d'une communauté à l'étranger, prise en étau par une double ambivalence: entre ancrage et mobilité, d'une part, entre ouverture et fermeture, d'autre part.

L'usage du mot diaspora induit trop souvent deux illusions. La première est de fondre une expérience géohistorique à d'autres, dans le cadre de comparaisons portant sur le rapport continu entre un peuple et un "référent origine", malgré la distance. La seconde est de permettre de postuler l'existence d'une communauté dont le nom, par synecdoque, est diaspora. Je désire aller au-delà de ces illusions pour exposer les structures élémentaires d'une diaspora et pour comprendre les différents états de la structuration de cette expérience (Dufoix, 2005). Des différents critères proposés par la plupart des auteurs, on peut en retenir quatre fondamentaux (propriétés ineffaçables ou typiques, en quelque sorte) directement en rapport avec l'emblème de la catégorie diaspora ${ }^{(4)}(c f$. tableaux 1 et 2);

- la population considérée s'est dispersée dans plusieurs lieux, en tous cas dans plus d'un seul territoire, non immédiatement voisins du territoire d'origine, sous la contrainte ; - le choix des pays et des villes de destination s'accomplit en conformité avec la structure des chaînes migratoires qui relient les migrants à ceux qui sont déjà installés dans le pays d'accueil ; ces derniers faisant figure à la fois de passeurs vers la société d'accueil et le marché du travail, et de gardiens de la culture ethnique ou nationale ;

- cette population s'intègre dans les pays d'accueil sans s'assimiler, c'est-à-dire conserve une conscience identitaire plus ou moins forte liée à la mémoire du territoire, de la société d'origine et de son histoire. Cela implique l'existence d'une vie associative assez riche, d'un "lien communautaire" (Hovanessian, 2005);

- ces groupes de migrants dispersés conservent et développent entre eux et parfois avec le pays d'origine, des relations d'échanges multiples.

Toutefois, dans cette analyse, je ne voudrais pas m'attarder sur le terme diaspora - notion ou concept - mais sur la manière dont je vais l'utiliser dans cette démonstration. Qu'est-ce à dire ? Simplement qu'il ne faut pas s'intéresser à la diaspora comme objet géographique, mais à la "diasporisation". "À la question "quoi" (...), il faut substituer la question "comment" pour restituer les processus par lesquels se forment les différents états de la structuration de cette expérience" (Dufoix, 2004). Les trois structures principales d'une diaspora sont le territoire d'accueil, l'ici; le "référent origine", là-bas; et l'ailleurs, c'est-à-dire l'ensemble des lieux de la dispersion. On comprendra que le "diasporé"(5) est en oscillation, il reste "disjoint", et ce qui donne une certaine cohérence à cet ensemble de disjonctions prend appui sur la filiation. Celle-ci prend l'aspect d'un lien entre ici et là-bas, ici et ailleurs. La cellule familiale, la religion et la sphère politico-économique constituent les relais de ce lien, la diaspora s'exprimant alors comme une volonté du lien resserré ( $c f$. schéma 1).

Pour illustrer mon propos, je pourrais évoquer un proverbe tamoul qui à lui seul résume les 
enjeux de la constitution d'un tel espace diasporique : "tout est mon pays, tout est ma parenté" ["Kanniyan Poongundran"]. En effet, on estime la diaspora tamoule à plus de 8,4 millions d'individus dispersés dans le monde (certes en plusieurs phases migratoires), soit un peu moins de $40 \%$ de ce que l'on appelle communément la diaspora indienne (Goreau-Ponceaud, 2008). La population tamoule étant établie un peu partout dans le monde, il devient impossible pour ses membres d'identifier leur territoire ("tout est mon pays"), car celui-ci s'étend peu à peu au globe dans son intégralité. La mobilité ne peut plus être qu'une mobilité du dedans, à l'intérieur d'un corps social devenu territoire ("tout est ma parenté"), constitutif d'un écoumène diasporique. De sorte que l'espace de la diaspora est partout et nulle part à la fois: "Ailleurs est convoqué ici mais, dans le même temps, il doit rester ailleurs parce qu'ainsi il garantit d'être ici" (Ma Mung, 1995, p. 170).

Ainsi, les membres de la diaspora tamoule habitent différentes mobilités; leur "bome n'est plus un lieu, mais plusieurs emplacements" (Bell Hook, cité par Urry, 2005, p. I53). Et l'on pourrait même dire que les Tamouls habitent le déplacement (Clifford, 1997). Ils combinent différentes manières ou façons " $d$ 'appartenir à" et de voyager "en deçà" et "au-delà" des frontières nationales. En effet, la totalité des personnes interrogées possèdent une famille éclatée sur au moins trois pays. Le plus souvent il s'agit du Royaume-Uni (98\%), du Canada (68\%) et des États-Unis (54\%). Mais si l'on prend en considération la famille élargie, ces destinations se diversifient et deviennent plus singulières : la Norvège (18 \%), les Pays-Bas (12\%), la Sicile (7 \%),

\section{Schéma 1}

\section{Les structures élémentaires d'une diaspora • Espaces, territoires et réseaux de diaspora}

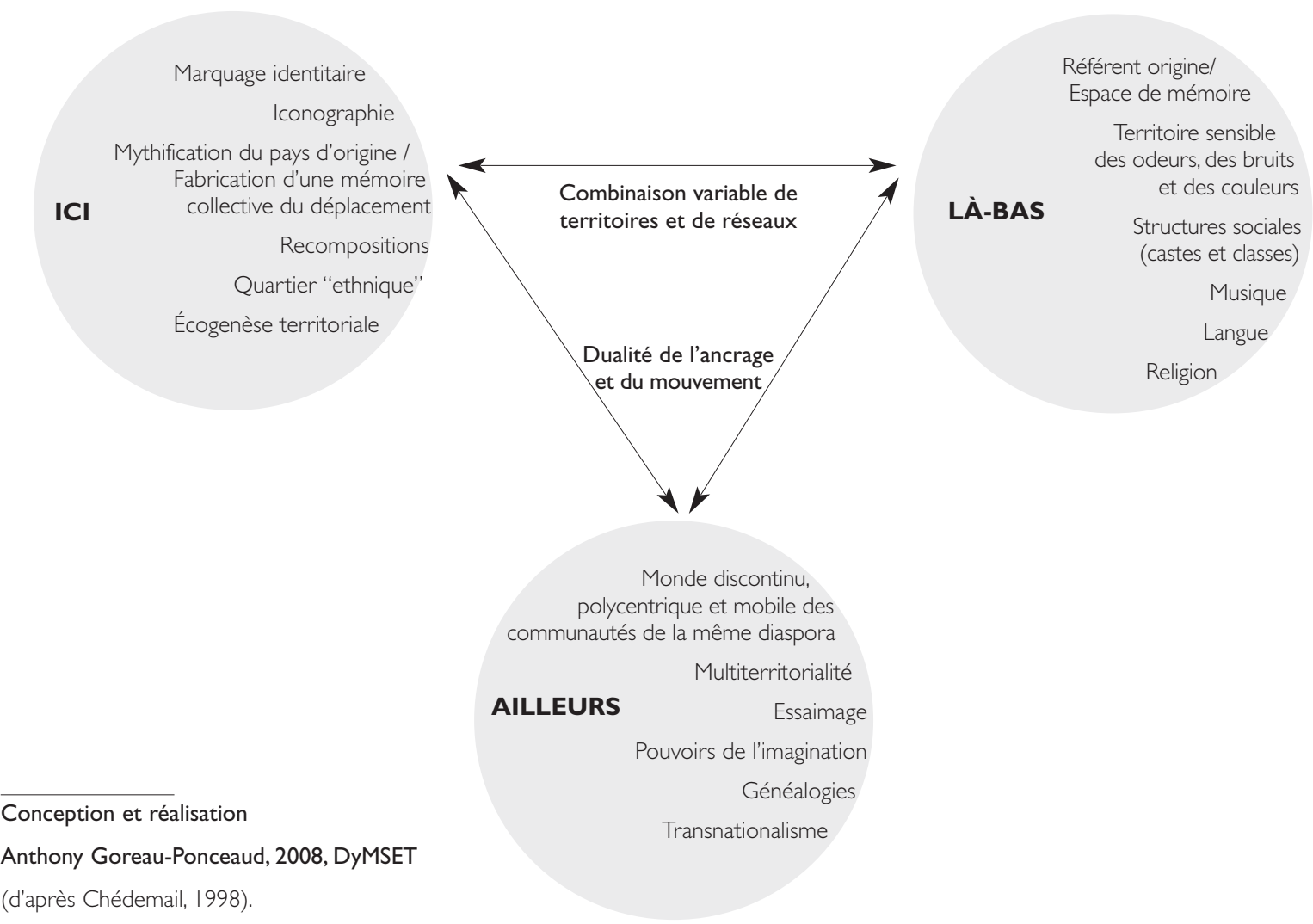


l'Allemagne (19\%), et la Suisse (22\%) font régulièrement partie de celles-ci pour l'Europe (78 \%), tout comme les Émirats Arabes Unis (12\%) (Abu Dhabi et Muscat plus précisément), le Viêt-Nam (4\%) et l'Australie (6\%) (cf. illustration 1).

C'est donc en faisant sauter le verrou national que les membres de la diaspora tamoule peuvent penser leur unicité. De plus, la filiation, ou la généalogie, permet aux membres de la diaspora tamoule de se représenter un espace commun qui est ici et là-bas. Si on ne peut pas être à deux endroits à la fois, et donc être de deux endroits à la fois (du lieu d'origine et du lieu de résidence), se projeter dans ce que Ma Mung nomme une "extraterritorialité” permet (Ma Mung, 1999) de se représenter un espace commun. Cette généalogie ("tout est ma parenté”) permet de reconstituer l'individu là où il est, en reliant de manière constante lieu de résidence et lieu d'origine. Cette performance, qui tient en la capacité de juxtaposer en un seul lieu plusieurs emplacements, relève de l'hétérotopie ; chacune des composantes de "ce lieu hors de tous lieux" est parfaitement localisable (Foucault, 1984).

Au regard de ces clarifications terminologiques, comment le tourisme s'insère-t-il dans ce rapport spécifique à l'espace qu'est la diaspora ? La diaspora peut elle être conçue comme l'archétype de l'habiter polytopique (Stock. 2006) ? Le "diasporé" est-il pour autant un touriste ? Comment concevoir les déplacements à l'intérieur de cet espace diasporique ? Ce retour au pays des origines constitue-t-il une pratique touristique ? Quelle est la part d'altérité liée à ce type de déplacement ? Si le tourisme, selon la définition donnée par Knafou et Stock, est un "système d'acteurs, de pratiques et d'espaces qui participent à la recréation des individus par le déplacement et l'habiter temporaire hors des lieux $d u$ quotidien" (Knafou et Stock, 2003, p. 931), de quelle manière procède cette rupture du quotidien pour ces populations qui se rendent en visite soit dans leur pays d'origine, soit dans un point "d'ancrage" constitutif de cet écoumène diasporique (Amérique du Nord, Europe, monde indianocéanique ou encore bassin caribéen) ? Les pratiques analysées en seconde partie vont permettre de concevoir ce rapport particulier à la diaspora et cette nouvelle façon de "construire de l'espace" par le tourisme.

\section{PratiQues touristiques : L'IDENTITÉ EN QUESTION}

\section{[Extrait $\left.\left.n^{\circ}\right|^{(6)}\right]$}

- Interlocuteur: "J'ai deux enfants. À chaque fois que je rentre ici, bien sûr on les emmène avec nous. Mais ça a l'air de les perturber. Hier matin, un de mes fils, le plus jeune, m'a demandé: "Je suis Indien ou Français ?’. Sa question m'a surpris, car pour moi elle ne se pose pas. Je suis les deux. Pourtant, je n'ai pas la double nationalité. J'espère que mes enfants pourront l'obtenir un jour, cela simplifierait beaucoup de choses... Mais je suis les deux. Mais pour moi, aller en France, ou revenir chez moi, c'est à chaque fois difficile. J'ai toujours à l'arrivée un temps d'hésitation, quant à l'attitude que je dois adopter, car même si c'est chez moi ici, j'ai parfois l'impression d'être considéré comme un touriste. Pourtant je suis né ici, j'ai ma famille qui habite ici, et j'entretiens des liens. Régulièrement on s'écrit, on se téléphone, j'envoie des photos. Mais bon, pour eux ça doit être encore plus difficile. Ils ne sont pas nés ici, ils n'ont pas de lien avec ce pays, sauf celui que je leur ai donné à la naissance, et celui que je leur donne par ces voyages. Ils savent aussi beaucoup de choses sur l'Inde, grâce à la famille, aux amis, à la TV, à internet..."

- Enquêteur : "Mais alors, comment définiriez-vous votre chez-soi et le leur?"

- Interlocuteur: "C'est comme qui dirait un peu partout et nulle part à la fois. C'est ici, mais aussi là-bas à La Courneuve et ailleurs, là où nous avons de la famille que nous visitons régulièrement, comme à Toronto, Berlin, Miami, Los Angeles, Basse-Terre, SaintDenis..."

Il semble que la position du "diasporé" soit paradoxale, ou tout au moins intermédiaire. Il apparaît n'être ni touriste ni résident per- 
manent ; pourtant, il peut jouir de droits spécifiques du fait de sa position particulière de membre de la diaspora indienne et être impliqué dans la vie citoyenne.

En effet, la diaspora indienne comprend de façon institutionnalisée trois grandes catégories qui correspondent, de façon schématique, à des cadres migratoires distincts : les People of Indian Origins (PIO), les Non Resident Indians (NRI) et les Overseas Citizens of India (OCI).

Les PIO sont des citoyens étrangers d'origine ou d'ascendance indienne. Trois cas de figure sont reconnus officiellement : toute personne qui a été détentrice d'un passeport indien ; toute personne dont les ascendants jusqu'à la troisième génération (arrières grands-parents) sont nés ou ont résidé de façon permanente sur le territoire de l'Inde indépendante ; l'épouse d'un citoyen indien ou d'un PIO. Cette catégorie désigne les Indiens qui ont émigré de manière forcée comme travailleurs sous contrats dans l'ensemble de l'Empire britannique et français, ainsi que les émigrés volontaires dont les migrations se sont faites au gré des préférences et des besoins personnels dans l'ensemble des pays du Commonwealth. Les PIO ont, depuis le début du $\mathrm{XX}^{\mathrm{e}}$ siècle, servi de levier à l'intelligentsia indienne pour rehausser l'image du pays et contribuer à son rayonnement culturel sur la scène internationale, en tant qu'ambassadeurs culturels de l'Inde dans leur pays de résidence.

La catégorie NRI, quant à elle, concerne des citoyens indiens détenteurs d'un passeport indien et résidant pour une période indéterminée à l'étranger. Plus largement, ce groupe rassemble tous les émigrants récents.

Enfin, les OCI sont des Indiens d'outre-mer qui ont émigré après le 26 janvier 1950 . C'est dans cette dernière catégorie que l'on retrouve un certain nombre de Français d'origine pondichérienne. Le statut d'OCI donne accès à la double nationalité (à l'exception de l'exercice de droits civiques et de l'acquisition de propriété agricole).

Les pratiques touristiques des “diasporés" que nous avons enquêtés sont les suivantes ( $c f$. tableau 3). Si le séjour est souvent long (29 jours en moyenne) et que $73 \%$ des personnes interrogées possèdent

Illustration I

\section{La diaspora tamoule, composante de la diaspora indienne}

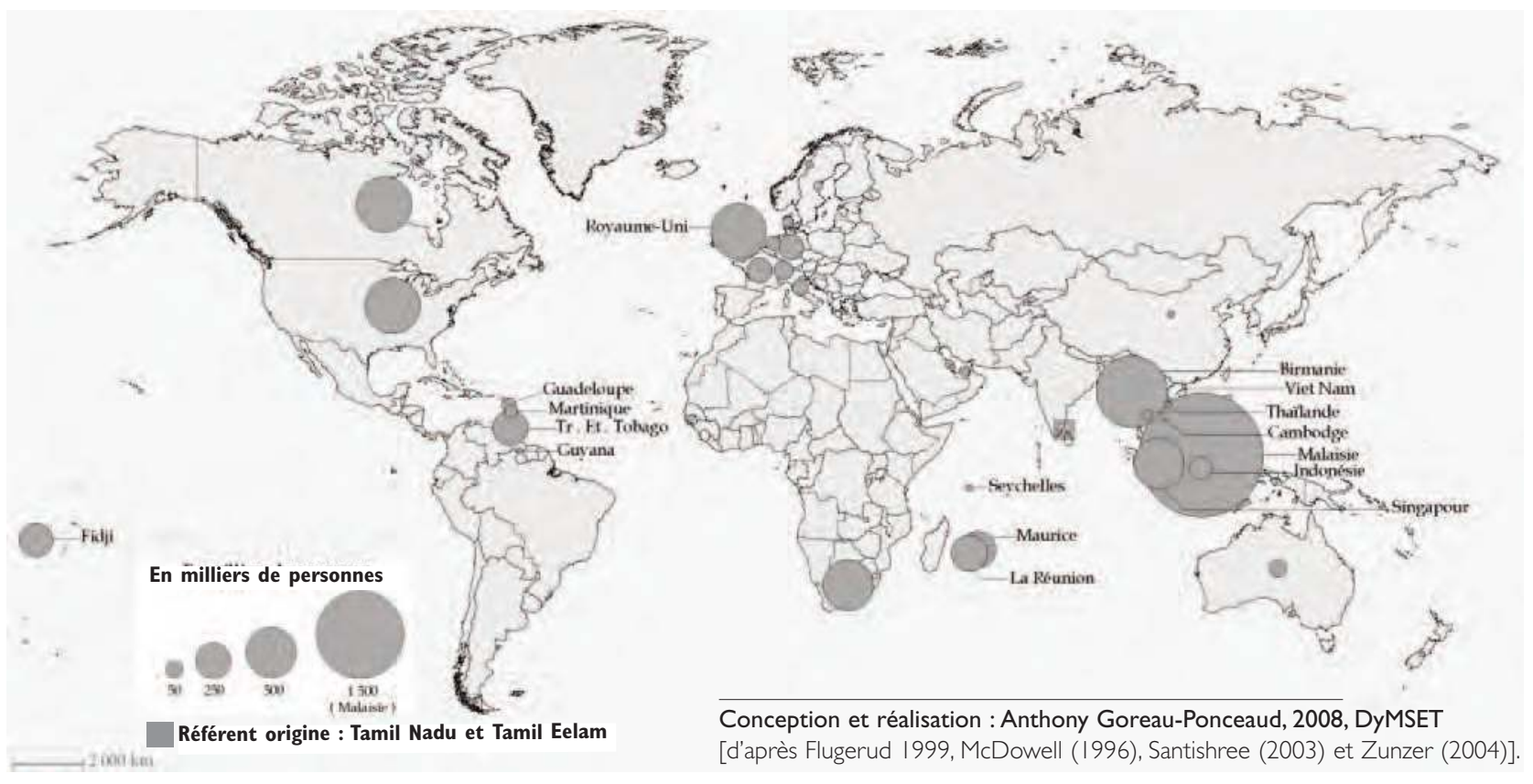


une maison de famille à Pondichéry, $69,2 \%$ de ces mêmes individus achètent, une fois sur place, un circuit au départ de Pondichéry. Il semble alors que, même si le nombre de séjours en Inde par an est supérieur à un, le "diasporé" ne soit pas fidèle à un territoire et à un domicile alternatifs. Certes, il répète en quelque sorte son désir de rester en contact avec la terre des origines, avec la famille, mais il reste cependant distant, ce qui lui confère cette situation ambivalente d'être un touriste chez lui. Dans tous les cas, il semble que cet éclatement, ou du moins cette tension spatiale, donne lieu à de nouvelles pratiques de mobilité axées sur la famille, que l'on pourrait apparenter, selon la typologie anglo-saxonne, à du tourisme dit visiting friends and relations (VFR), d'une part, et analyser à travers le prisme du transnationalisme, d'autre part.

Cette forme de tourisme nécessite des adaptations et innovations de la part des acteurs du tourisme, qu'ils soient institutionnels on non. De nouvelles offres se font jour à destination des familles originaires
d'Inde installées en France : on assiste à la multiplication des circuits de pèlerinage.

\section{Des pratiques mémorielles} aux pratiques de "recréation"

Plus généralement, les sites historiques du sud de l'Inde - dont certains inscrits au patrimoine mondial de l'Unesco tels que Mahabalipuram et les temples Chola du Tamil Nadu -, les lieux de départ ou d'émigration (Pondichéry, Karikal et Chennai) et les lieux faisant partie de l'histoire nationale de l'Inde figurent parmi les étapes de ces circuits développés en France, mais également en Inde, par des agences parfois détenues par des Indiens de la diaspora (c'est le cas de trois agences à Pondichéry). On assiste également, depuis 2008, à la création de maisons d'hôtes à Pondichéry, par des Français originaires de Pondichéry, dans le but de structurer ce tourisme de diaspora, en provenance d'Europe et des ÉtatsUnis principalement.

Par ailleurs, l'un des défis de la campagne "Incredible India" (nom

Tableau 3

Pratiques de la population interrogée à Pondichéry en juin 2010 (effectif 52 personnes)

Hommes

$61,5 \%$

Femmes

Durée moyenne du séjour en Inde

Possèdent une maison de famille

Nombre de séjours par an en Inde en moyenne

Personnes ayant contracté un circuit au départ de Pondichéry
$38,5 \%$

29 jours

$73 \%$

I,7

$69,2 \%$ officiel du site internet du ministère du Tourisme indien) est d'associer de nombreuses images du pays avec l'enracinement, la tradition et le passé, afin de stimuler et d'encourager le tourisme de diaspora, qui est particulièrement sensible à ces figures mnémoniques. Ces pratiques touristiques sont aussi, pour ces personnes, un moyen de mesurer les écarts entre l'Inde et leur lieu de résidence. Les membres de la diaspora indienne se rendent bien compte, à ce moment précis, des contrastes qui existent entre être Indien en Inde et être Indien en France, d'où parfois un sentiment paradoxal d'être un touriste chez soi.

L'Union indienne a également adopté une approche marketing très moderne, fondée sur des publicités diffusées sur Youtube. Dès 2007, le secrétaire du ministère du Tourisme, Shri Shilabhadra Banerjee, a créé une chaîne sur le web [www.youtube.com/India] qui est relayée par les médias sociaux. Lors du lancement de cette opération, il y avait dix-huit films à télécharger. Pour le ministère du Tourisme, la diffusion de ces films via les médias sociaux permet aux potentiels voyageurs de la diaspora (mais pas exclusivement) d'avoir une première expérience avec l'Inde. C'est également un moyen de montrer au monde que l'Inde a fait des progrès depuis son indépendance, et de gommer ainsi certains poncifs qui pèsent encore sur ce souscontinent. 
Ces pratiques touristiques sont donc marquées par la nostalgie, et les lieux fréquentés sont rattachés, de manière non exclusive, tant à la mémoire familiale qu'à l'histoire nationale.

[Extrait $\left.n^{\circ} 2\right]$

- Enquêteur : "Pourquoi venezvous à Pondichéry?"

- Interlocuteur : "Je viens d'abord pour voir la famille. Mes parents ont une maison ici. Je viens chaque année passer du temps dans la ville où je suis né. Nous venons, ma femme et moi, passer du temps ici. Nous essayons de venir à chaque grandes vacances, avec les enfants. Généralement nous restons pendant quatre semaines. C'est la période où il y a le plus de monde à Pondichéry. Il y a beaucoup de touristes!"

- Enquêteur: "Vous ne vous considérez pas comme un touriste?"

- Interlocuteur : "Oui et non. Non car je viens voir ma famille, c'est chez moi ici. Mais en même temps on est mobile, on fait des choses que l'on ne fait pas à Paris, surtout depuis que les enfants sont grands. Voir la famille, pour eux, c'est un peu ennuyant. Pourtant, on prend plaisir à leur montrer les lieux de notre enfance, le patrimoine de cette ville qu'ils ne connaissent pas. Vous savez, c'est la "French Touch" qui fait venir tout ce monde ici. Bien sûr il y a l'Ashram, Auroville... mais... de toutes façons, concernant nos enfants, j'ai l'impression qu'ils préféreraient qu'on aille voir nos cousins qui sont installés à Los
Angeles. Pourtant, à chaque fois que l'on vient rendre visite à la famille, on en profite pour découvrir d'autres États : le Kerala par exemple. L'an dernier, on a même profité de notre séjour pour aller aux îles Andaman. On avait pris un billet depuis Chennai. Quelquefois, on fait même appel à une agence de voyages qui est détenue par un compatriote. Il organise des voyages pour les Français de souche qui veulent connaître le passé de la ville. D'une manière générale, nous on évite de s'inscrire aux formules proposées par les offices de tourisme. Cela ne nous convient pas".

Cet extrait d'un entretien mené à Pondichéry en juin $2005^{(7)}$ nous éclaire sur le type de pratique de mobilité des individus originaires du Tamil Nadu ou du territoire de Pondichéry. Les critères de définition de cette pratique de mobilité sont ici les suivants : elle est choisie, lointaine et familière. Cependant, pour les enfants de cette famille, les lieux appartiennent au "hors quotidien" (cette ville qu'ils ne connaissent pas), si bien que pour cette génération, cette pratique de mobilité s'apparente au tourisme et non au loisir ou à la simple visite à la famille. Qui plus est, le sentiment d'altérité n'est pas moindre pour eux qui arrivent, rencontrent leurs ascendants et découvrent, parmi ces “autochtones" qui n'ont pas émigré, une manière d'être différente de celle des membres de leur propre famille ou communauté.
Comment envisager - voire "dévisager" si on voulait utiliser un terme plus fort - tous ces "étrangers" et la vie parmi eux ? La réponse à cette interrogation implique, pour les membres de la diaspora (et particulièrement pour la seconde génération), la mise en œuvre de compétences sociales censées innover ce rapport à l'autre. Car, pour eux, cette visite de famille est une véritable découverte des leurs (vu comme des autres), qui permet de déplacer les frontières de l'altérité jusqu'aux confins de la diaspora (des autres acceptés comme les leurs). Pour les parents, ce temps limité du retour est certes l'occasion de réactiver des liens, mais surtout de se reposer et de découvrir d'autres lieux : des modalités qui participent à la "recréation touristique" (Stock, 2003). Par ailleurs, on peut noter que les pratiques ne sont pas les mêmes pour les membres de la diaspora et pour la population locale. Pourquoi ? La réponse est à rechercher à l'aide du concept d' "habitus touristique" et de "capital de mobilité", qui ne sont pas de même nature (Ceriani-Sebregondi, 2006 ; Stock, 2005). Ainsi, ce tourisme des racines ou généalogique n'est pas seulement une manière de maintenir les liens avec le groupe ou le "référent origine" afin de transmettre la mémoire ou l'histoire collective aux nouvelles générations. Il s'accompagne de ce que l'on a appelé des "pratiques déroutinisantes” (Stock, 2005).

En effet, la majeure partie des 
personnes interrogées effectuent régulièrement des circuits. Les destinations fréquentées sont multiples et correspondent à des finalités variées. Les îles Andaman apparaissent comme une destination privilégiée, de même les hauts lieux de l'hindouisme. La diaspora thématise ainsi l'image de certains sites touristiques et permet aussi l'élargissement de la saison. C'est ainsi que la ville de Pondichéry est très fréquentée durant l'été, alors que la saison touristique au Tamil Nadu s'étend de décembre à mars.

En somme, ce tourisme de diaspora qualifie des lieux, des quartiers par de nouveaux usages ; il subvertit des lieux existants et devient une activité supplémentaire dans ces exterritorialités constituées par la diaspora indienne. Il peut également être institutionnalisé dans l'espoir d'opérer une diversification de l'offre et d'attirer des investissements.

\section{Discover India. Discover yourself. Vers une institutionnalisation des pratiques touristiques en diaspora}

Malgré ces efforts et ces nouvelles pratiques, l'Union indienne apparaît en marge des destinations touristiques mondiales. En 2008, selon le rapport du ministère du Tourisme indien, seulement 5,37 millions de personnes s'étaient rendues en Inde ${ }^{(8)}$. L'Union indienne arrive seulement en $41^{e}$ position des destinations touristiques mondiales, et ne polarise que $0,58 \%$ des flux touristiques internationaux. Nous sommes ici bien loin des 562,92 millions de personnes constituant les flux du tourisme intérieur. Malgré tout, la société indienne est en cours de "touristification", le nombre de touristes se rendant en Inde depuis 2002 est en constante augmentation (+140\%). L'un des éléments permettant d'expliquer ce saut quantitatif est l'effort consenti par le gouvernement indien depuis les années 2000 pour stimuler le tourisme de diaspora. Il a en particulier créé le High Level Committee on the Indian Diaspora ${ }^{(9)}$ dont l'objet est de recenser la diaspora indienne et d'établir son rôle dans l'économie de l'Inde. Ce comité a préconisé la création de cadres institutionnels pour organiser et encadrer la politique de développement touristique dans la mesure où la diaspora peut contribuer à la croissance du tourisme en Inde. Ainsi, face à l'ouverture économique post-1991, le comité est intimement convaincu qu'il est temps, dans le contexte de globalisation, d'associer de diverses manières les membres de la diaspora indienne à ce renouveau économique, tout en ravivant leur sentiment d'appartenance à l'Inde. C'est aussi dans ce cadre que des événements comme le Pravasi Bharatya Divas ${ }^{(10)}$ peuvent être considérés comme des espaces dans lesquels les définitions de l' "indianité" et les questions d'authenticité et de continuité sont mises à l'épreuve.

Certains États de l'Union indienne ont également développé des initiatives pour encourager ce tourisme de diaspora et, plus particulièrement, ce tourisme dit des "racines" (roots tourism selon la terminologie employée par l'État de l'Uttar Pradesh). C'est, par exemple, le cas de l'Uttar Pradesh, État de la plaine gangétique qui a connu au cours du XIX ${ }^{e}$ et $\mathrm{XX}^{\mathrm{e}}$ siècle une émigration massive de travailleurs engagés ou coolies vers les colonies britanniques. Pour permettre aux descendants de ces engagés de découvrir leurs "racines", le ministère du Tourisme de cet État, au cours des années 2000, a mis en place un programme dont le nom est significatif : "Discover your roots scheme". Ce programme aide les personnes d'ascendance indienne à retrouver leur lieu de naissance (contre 150 dollars et trois mois d'attente en moyenne). Ce programme a été développé dans l'hypothèse qu'il pourrait intensifier le tourisme dans l'État et renouveler une offre déjà diversifiée (cet État concentre $11 \%$ des flux de touristes étrangers et 22,2\% des flux de touristes nationaux). Il est aussi fondé sur l'hypothèse qu'un habitant sur six vivant dans le monde est Indien, et qu'un Indien sur six habite ou est originaire de l'Uttar Pradesh. Ainsi, même si pour les cent premières personnes, le service est gratuit, il pourrait être une source importante de revenus. Néanmoins, au-delà de ces innovations dans l'offre touristique, le programme n'est pas un succès et 
n’a pas été copié par les autres États à l'origine d'une forte migration durant la même période, comme le Tamil Nadu ou le Gujarat par exemple. En revanche, de tels programmes existent dans des pays à forte émigration indienne, à l'instar de l'̂̂le Maurice (où la population originaire du sous-continent représente $70 \%$ de la population totale) qui a également mis en place un programme "tracing roots".

\section{DES PRATIQUES IDENTITAIRES AUX PRATIQUES TOURISTIQUES}

S'il existe véritablement de nouvelles pratiques touristiques prenant corps et place au sein de la diaspora tamoule-indienne, cette configuration diasporique nous oblige à réviser certains poncifs sur les relations entre Nord et Sud, entre foyers émetteurs et récepteurs des flux touristiques. Ceuxci deviennent en effet multidirectionnels. En cela, les diasporas suscitent une nouvelle prise de possession du monde par le tourisme qui repose sur des pratiques mettant sur le devant de la scène des lieux touristiques émergents dans des espaces initialement peu "touristifiés”. En effet, chaque point d'ancrage de la diaspora indienne est susceptible d'être pensé comme un site touristique. Dès lors, le tourisme de et en diaspora s'exerce à l'échelle mondiale. Un seul continent, au regard de nos entretiens, apparaît complètement ignoré de ces pratiques : l'Amérique Latine. De même l'Afrique, hormis la partie australe et orientale, semble marginalisée. Dès lors, ces pratiques créent des saillances dans l'espace diasporique. C'est d'ailleurs de cette manière qu'une centralité minoritaire indienne à Paris, le quartier de La Chapelle (compris entre les $\mathrm{X}^{\mathrm{e}}$ et XVIII ${ }^{\mathrm{e}}$ arrondissements) est devenu un lieu polarisant des flux touristiques conséquents lors d'événements particuliers.

Dans Les Cadres sociaux de la mémoire, Halbwachs établit que la pensée sociale est essentiellement une mémoire et que, pour durer, celle-ci doit s'attacher à quelques points du sol (Halbwachs, 1994). C'est ainsi que l'on a assisté, à partir des années 1980, dans ce nœud que constitue la métropole parisienne, à la construction d'un territoire ethnique dont les symboles et l'iconographie sont les marqueurs. En faisant appel à la mémoire du "référent origine", ces marqueurs déterminent un ancrage territorial qui sculpte les contours d'une "communauté imaginée" (Anderson, 2002). De sorte que le local devient "lieu de production d'identités communautaires sur le modèle de celles qui prévalaient au pays, par l'intermédiaire de pratiques ou d'institutions assurant la pertinence actuelle des cadres d'hier dans leur forme d'ici et maintenant" (Dufoix, 2003, p. 82).

Au sein de ce quartier, on recense deux temples hindous, complétés d'un troisième situé dans le XIX arrondissement et de onze autres dans les communes de la banlieue nord et est de Paris. L'un est dédié à Ganesh, l'autre à Maryammâ. Le plus important des deux, le temple ou association Sri Manicka Vinayakar Alayam (dédié à Ganesh), fondé en 1985, s'affiche comme le "temple hindou de Paris" du fait de sa stratégie promotionnelle (site internet, production et diffusion de vidéos, publicité), parce qu'il célèbre toutes les fêtes du calendrier hindou et en particulier qu'il organise, depuis 1996, le Festival de Ganesh ou Ganesh Chaturthi. À cette occasion, les Hindous d'Île-de-France organisent un défilé où le char du dieu à tête d'éléphant est promené dans les rues voisines du temple, situé au 72 rue Philippe de Girard. Au cours du trajet, le cortège, composé de danseuses portant sur leurs épaules des arceaux de plumes de paon et sur leur tête des pots de terre cuite, passe devant des tas de noix de coco qui sont alors brisées et distribuées. Vient ensuite le char de Ganesh, mesurant près de 5 mètres de haut et tiré par des hommes à l'aide de deux cordes de vingt mètres de long, puis celui de Murugan, tiré par des femmes (cf. illustration 2).

C'est à la suite d'un miracle que cette fête en l'honneur de ce dieu est organisée tous les ans dans les rues de Paris ; la date de la célébration, fixée en Inde par des astrologues, varie entre les mois d'août 
et septembre ${ }^{(11)}$. En effet, le 21 septembre 1995, en Inde comme dans l'ensemble des pays du monde où résident des Indiens, les dieux se sont manifestés ouvertement : ils ont bu le lait qui leur avait été offert en oblation. "Le miracle put être constaté partout où se trouvaient les statues de Ganesh et des divinités associées à Shiva: que ce soit dans les temples ou chez les particuliers et aussi bien en Inde que dans le monde entier; partout, en fait, où il y avait des dévots ou même des simples curieux qui voulaient en faire l'expérience" (Vidal, 1997, p. 884). Ce phénomène ne dura que le temps d'une journée, mais a suffi à bâtir, du côté du "référent origine", une historiographie de la diaspora. Cet événement, en plus de constituer un rituel public qui renvoie à l'idée d'une délimitation sanctifiante de l'espace et du temps, est un moment de convivialité et de sociabilité qui facilite le rapprochement entre les membres de la diaspora, ce qui est confirmé par l'importance, à ce moment là, des séjours en France des Indiens chez les parents, amis ou membres de la famille élargie.

En 2008, lors de mes dernières enquêtes dans ce quartier, la fête avait rassemblé 15000 personnes. Parmi elles, j'ai pu interroger des Indiens venant d'Allemagne, de Suisse, d'Italie, de Guadeloupe et de La Réunion. Bien sûr, ces personnes profitaient de visites au sein de leur famille pour venir assister à cette fête et visiter Paris.
Ainsi ces temples, en tant que nouveaux lieux de mémoire, deviennent peu à peu des lieux touristiques. Ceux-ci, en tant que monuments, sont constitutifs d'un patrimoine qui développe le sens culturel d'une présence transnationale imaginée, en développant un métarécit biographique dans et à travers les flux globaux. L'implantation d'un tel lieu répond aussi à des exigences. Dans ce processus, la nostalgie joue un rôle indéniable. Elle se traduit notamment par la reproduction d'une institution culturelle familière dans un monde étranger, par le truchement d'un style architectural rappelant le gopuram (tour au plan rectangulaire surmontant les portes des temples de style dravidien), ou l'intérieur du temple du village d'où l'on a migré (cf. illustration 3). Il s'établit là des rapports particuliers entre architecture, mémoire et identité collective: "le monument en perpétuant les réminiscences et en focalisant les caractères communs d'une collectivité, est incubateur et paradigme des syntagmes identitaires" (Noppen et Morisset, 1997, p. 35).

Ce patrimoine a pour ambition de sauver l'histoire diasporique en même temps qu'il la raconte. De plus, ces lieux du sacré, qui sont visités, appropriés et pratiqués en France, se situent eux-mêmes dans un ensemble diasporique, en tant que pôles structurés par des circuits de voyage formant ainsi les "axes latéraux de la diaspora"

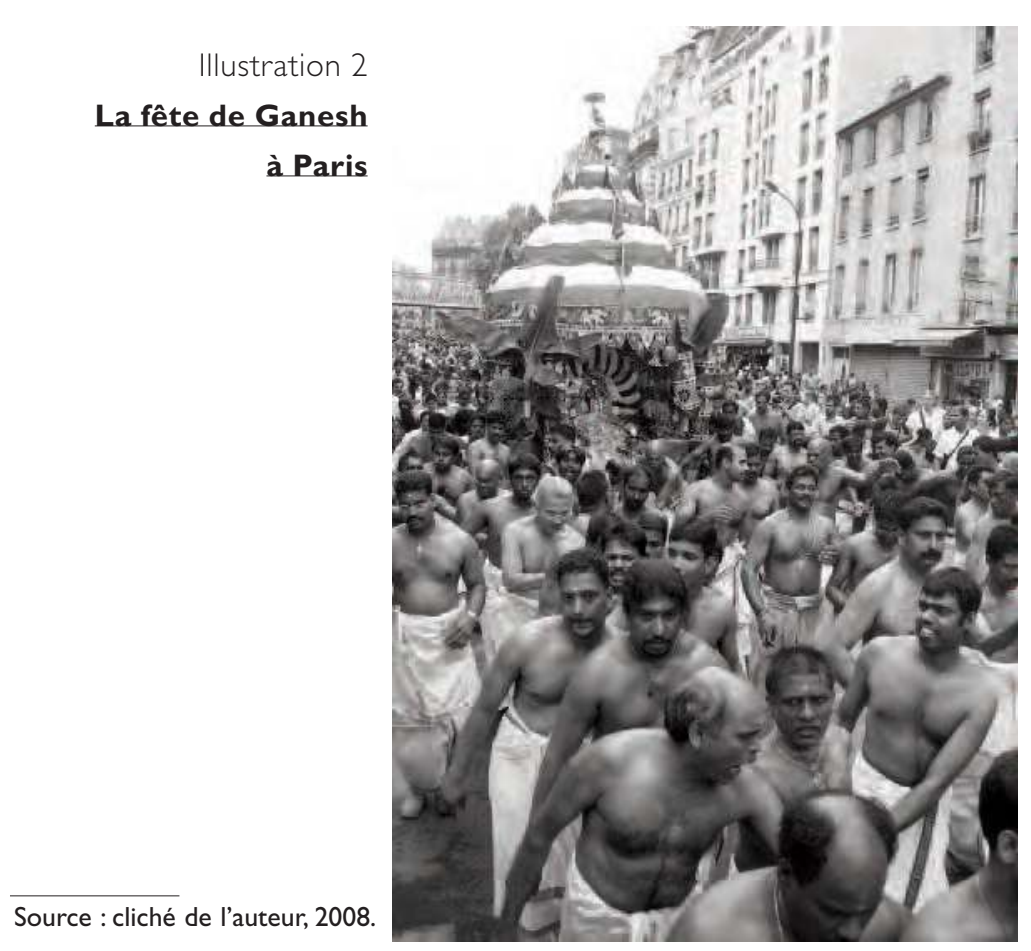


(Clifford, 1997, p. 269). Parmi les personnes interrogées lors de cet événement, certaines ont organisé via les médias sociaux la visite de plusieurs temples en Île-de-France. Au travers des blogs, certains ressortissants indiens proposent des services de guidage à destination de personnes originaires elles aussi du sous-continent ${ }^{(12)}$. Plus surprenant, il existe des visites à destination quasi exclusive de la diaspora.

C'est ainsi que j'ai rencontré en 2009, à La Courneuve, un groupe d'Anglais originaires d'Inde, venus visiter le temple de Shiva. Ils avaient acheté à Southall Broadway leur circuit organisé mêlant pratiques "déroutinisantes" (visite des monuments clés de la capitale, à l'instar de la Tour Eiffel et de l'Arc de Triomphe) et mémorielles.

Dans un autre registre, une agence de voyages de Pondichéry, Souvenir Travels, proposait dans son tour d'Europe et de Dubaï (au mois de mai 2010) d'une durée de vingt jours $^{(13)}$, la visite à Londres du temple hindou Swaminarayan. Les lieux visités dans la capitale parisienne faisaient la part belle au quartier de La Chapelle. Cependant, d'après Suresh Somani, le directeur de l'agence, “il faut encore que nous affinions l'offre, car mes clients ont trouvé que deux jours de visite à Paris c'était trop

\section{Illustration 3}

\section{Homologies architecturales}

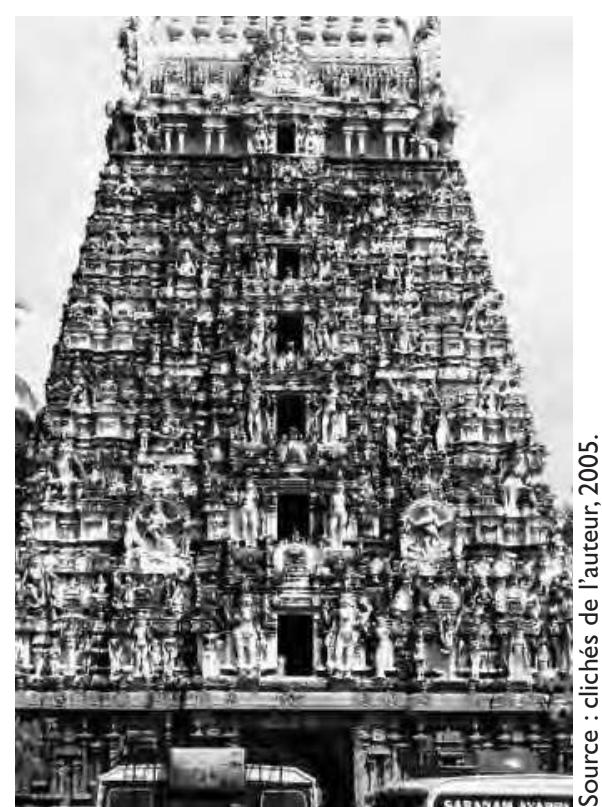

Entrée du temple de Shiva

à Villenour (territoire de Pondichéry)

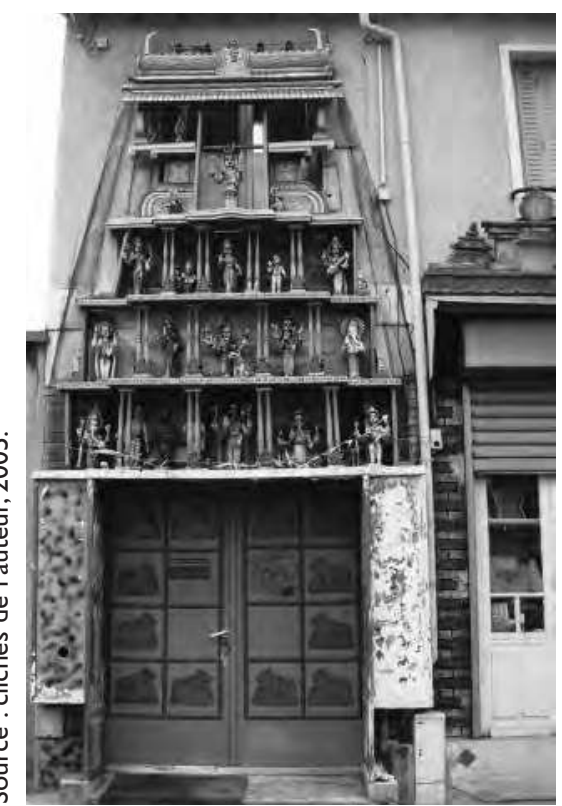

Entrée du temple de Shiva

à La Courneuve (93) long. Il n'y a pas de temples hindous aussi importants qu'à Londres, et la visite des musées ne les intéresse pas. C'est pourquoi le troisième jour était consacré à la visite du parc Eurodisney" (entretien du 14 juin 2010).

Par ailleurs, là où le lien entre la diaspora et les pratiques touristiques de et en diaspora apparaît le plus important, dans cette offre touristique structurée en Inde pour des Indiens, c'est quand arrive le moment des repas. En effet, que ce soit à Paris, à Londres, à Genève, à Innsbruck, à Vienne, à Venise, à Florence ou encore à Rome, l'ensemble des repas sont pris dans un restaurant indien (le Salaam Namaste, à Londres, ou le Jaipur Palace, à Paris, par exemple). "L'ensemble de mes clients apprécient seulement la nourriture indienne végétarienne. Je prends donc soin, avec mes correspondants, de trouver de bons restaurants indiens dans les lieux que nous visitons" (entretien du I 4 juin 2010).

Les lieux visités par cette clientèle indienne se confondent ainsi avec ceux de la diaspora indiennetamoule. Les destinations les plus fréquentées sont la Malaisie, l'Île Maurice, le Sri Lanka, et les Émirats Arabes Unis ${ }^{(14)}$. Toutes sont des pôles importants de la diaspora indienne et plus particulièrement tamoule. "Je pense organiser un prochain voyage aux États-Unis. Après l'Asie, l'Océan indien et l'Europe, mes clients sont très 
friands de ces destinations. Il n'y aura pas de problèmes pour trouver des restaurants indiens et de beaux temples à visiter. En Afrique, un seul pays les attire, c'est l'A frique du Sud. Ils m'ont demandé d'organiser un voyage pour dans deux ans là-bas" (entretien du 14 juin 20 I0). Là encore, l'ouverture à de nouvelles destinations se fait en fonction de l'importance diasporique, ce qui est particulièrement flagrant dans le cas du continent africain où seule l'Afrique du Sud est mise en tourisme et balisée pour ces types de flux.

De son côté, le quartier de La Chapelle à Paris, en tant que concentration commerciale, est également marqué par des usages touristiques. Pour les Indiens et Tamouls, "il y aurait retour aux sources et à l'endotique, au "comme chez soi" et au "comme autrefois". Pour les autochtones, il y aurait dépaysement, goût de l'exotique, recherche de l'ailleurs, ouverture sur le monde" (Raulin, 2000, p. 22). C'est ainsi que de multiples balades sont proposées par des guides de voyage (le Routard par exemple), par des guidesaccompagnateurs - Poonam Chowla, ancienne guide travaillant pour le ministère du Tourisme indien, propose des visites de 90 minutes du quartier au prix de 25 euros par personne -, mais également par des sites internet liés au développement touristique soit de la ville de Paris, soit de la région Île-de-France. C'est ainsi que nous pouvons lire, sur le site tourisme.paris.fr, "Dans les XVIII et $X^{e}$ arrondissements, l'Afrique de la Goutte d'Or et l'Inde du faubourg Saint-Denis vous invitent à une balade dans le Paris d'ailleurs" (15). Ce binôme tourismecommerce n'est pas ici source de conflits. Au contraire, comme le souhaite le gouvernement de Delhi, cette présence transnationale permet de diffuser l'image et l'imaginaire de l'Inde. La diaspora est le principal instrument du rayonnement culturel et politique de l'Inde à l'étranger. L'Inde s'appuie sur ce soft power, sur ces "ambassadeurs", pour rehausser l'image du pays.

Cette culture de la mobilité que nous venons de décrire engendre paradoxalement une quête de l'enracinement et de l'enfermement. Le festival de Ganesh est un moment authentique car vécu sur le mode de relations elles-mêmes authentiques (du moins dans l'imaginaire). Cette authenticité renvoie aux relations de confiance. Cette forme de pratique touristique évoque le repli sur soi. Ce n'est plus uniquement l'espace qui définit le tourisme, ce sont aussi d'abord et surtout les images du refuge, de l'enracinement qui définissent un imaginaire et fondent une expérience touristique. Sans tomber dans la "nissonologie" (Moles, 1982), il semblerait bien que La Chapelle soit pensée de l'intérieur comme une sorte "d'île sur le sol de laquelle il est possible de reconstituer un univers symbolique et familier" (Dufoix et Foucher, 2007, p. 431).

La valeur de l'authenticité semble influencer les pratiques touristiques et culturelles de et en diaspora et contribuer au renouvellement des lieux fréquentés et à leur permanence. D'ailleurs, au sein de cette centralité minoritaire, nous pouvons recenser onze agences de voyages qui proposent des circuits mais surtout des vols à destination du territoire d'origine ${ }^{(16)}$. Ces agences ont pour particularité d'être détenues par des personnes originaires du monde indien, de même que les personnes qui y travaillent et la clientèle visée par les offres commerciales. Ici, l'authenticité rime avec confiance et sincérité. Le tourisme diasporique ne permet pas seulement de maintenir du lien entre les pôles de la diaspora, il contribue également à la découverte de la terre des ancêtres, il permet de renouer les fils de la généalogie familiale et de faire l'expérience concrète du pays d'origine. Cette forme particulière de voyage se situe, pour mes interlocuteurs, entre l'entreprise identitaire - une sorte de voyage initiatique qui vient donner le change au déracinement et satisfaire les attentes mémorielles et la curiosité, en particulier pour la génération née en France - et récréative - il s'agit également de prendre du plaisir, de rompre avec le quotidien. Au final, ces touristes tissent un type de relation alter- 
natif à la société qui donne aux sites pratiqués une autre valeur et une autre fonction.

\section{CONCLUSION}

LE TOURISME DE ET EN DIASPORA : UNE PRATIQUE ÉDIFIANTE

Avec la mondialisation, la demande touristique a connu de fortes mutations qui imposent aux destinations et aux territoires une sérieuse remise en cause; œuvrer à la visibilité et la lisibilité des destinations semble donc être l'enjeu de toute politique d'attractivité touristique. La diaspora indienne peut devenir un levier essentiel au développement touristique de l'Union indienne et contribuer à son rayonnement international : en devenant un support de communication institutionnalisé par l'État central, en étant un acteur du secteur du tourisme, et en mobilisant des consommateurs à la recherche d'un passé qui permette de constituer un présent. Certes, les diasporas ne sont pas un acteur nouveau, mais la mondialisation les favorise doublement : en créant un contexte d'action réticulaire et en renforçant l'attraction des racines culturelles. Cependant, ces pratiques touristiques, développées au sein d'un espace diasporique et par une diaspora, nous rappellent également que l'identité n'est pas une donnée, ni une ontologie, ni une évidence, ni un fait de nature, mais une construction où jouent avant tout les représentations, les images, la mémoire. En cela, l'utilisation de la terminologie "roots tourism" par les divers acteurs institutionnels et chercheurs apparaît bien maladroite. Ce que nous offrent ces pratiques, c'est bien la pensée d'un possible divorce entre identité et territoire. Tout au moins, ces pratiques nous invitent à renouveler le concept de territoire. Cela pose évidemment la question de savoir si et comment cette expérience de la mobilité touristique va affecter l'individu et sa construction identitaire, comment elle peut transformer son rapport au monde, aux autres et à lui-même.

Ces pratiques touristiques permettent également à l'individu de se construire en tant que membre de la diaspora tout en construisant l'espace de la diaspora. Il y a donc là une relation de réciprocité, une sorte de bouclage récursif entre ces pratiques et l'espace de la diaspora. Ces pratiques servent à unifier les lieux et les différents espaces sociaux indiens dans lesquels les identités transnationales ont été façonnées et entretenues. Le tourisme, forme particulière de déplacement, pourrait être analysé comme un moyen par lequel les relations transnationales entre les différents points d'ancrage de la diaspora indienne sont maintenues et renforcées. En cela, le tourisme de et en diaspora est édifiant ; il est un acte par lequel les membres de la diaspora indienne se constituent socialement comme sujet et prennent conscience de leur appartenance à cet espace particulier qu'est la diaspora.

Cette recherche permet d'entre- voir l'importance des pratiques touristiques dans les études diasporiques, longtemps marginalisées ${ }^{(17)}$. L'analyse du tourisme de diaspora s'inscrit dans un courant qui fait de la mobilité l'un des fondements des processus actuels de formation des cultures (Urry, 2005). Aussi, comme l'a avancé Clifford, dans un monde continuellement "en route" (Clifford, 1997), le "diasporé" apparaît comme une figure de la postmodernité. Les métaphores du retour à la maison, de la quête identitaire, de la famille forment une grammaire composite du phénomène de tourisme en diaspora. Mais pas seulement, car cette étude montre également la vacuité de la distinction entre migration et circulation, qui se fonde sur le caractère permanent ou temporaire des différents mouvements (Stock, 2006); car ces pratiques qui prennent corps au sein de la diaspora indienne passent progressivement du statut d'éléments permettant le maintien des liens familiaux à des pratiques de recréation où la fonction "déroutinisante" est importante.

Au final, est-ce que le terme "pratiques touristiques" convient pour décrire ces formes de mobilités ou bien faut-il trouver autre chose dans le "spectre de recréation" (Stock, Violier et Duhamel, 2009) ? 
(I) Notons que le terme "tamoul" renvoie d'abord à un groupe ethnolinguistique du sous-continent indien. Le tamoul est une langue du sud de l'Inde, dominante au pays tamoul (ou Tamil Nadu) et au nord Sri Lanka, appartenant à la famille dravidienne qui se distingue des langues du nord principalement indoeuropéennes (hindi, gujarati, bengali, ourdou....). On parle donc de Tamouls à propos de la population tamoulophone.

(2) Parmi les 284 enquêtés d'l̂e-de-France, 22 I d'entre eux étaient originaires de Sri Lanka, 22 de la Réunion et de Maurice, 12 des Antilles et 29 de Pondichéry. Parmi les 239 personnes interrogées en Inde du sud (au Tamil Nadu, dans les districts de Ramanathapuram et de Naggapatinam, et dans le territoire de Pondichéry), I 64 étaient des Français d'origine pondichérienne, 22 des Tamouls ayant de la famille installée en Île-de-France et 53 des réfugiés sri lankais ayant fuit par bateau le Sri Lanka.

(3) Sur un échantillon composé dans sa totalité de 523 personnes, seules 52 personnes, en majorité originaires de Pondichéry, ont été interrogées et interviewées, en Inde et en France, au sujet de leurs pratiques touristiques; 215 l'ont été concernant leurs pratiques de double résidence. Ces questionnements sur les relations entre pratiques touristiques et diaspora, démarrés durant mon doctorat, font l'objet de nouvelles enquêtes depuis 20 I0. Je mène également cette analyse en partenariat avec des étudiants de première et seconde année de I'IUT de PérigueuxBordeaux 4, effectuant leur stage dans des agences de voyages de Pondichéry, sur la manière dont le secteur marchand réagit face à ces nouvelles demandes touristiques. Cette contribution reflète donc certains résultats acquis lors de ces investigations.
(4) Si l'objet diaspora a vocation désormais "universalisante" (Médam, 1993), il a été longtemps réservé aux Juifs pour désigner leur dispersion après la destruction du Temple, faisant de cette expérience un archétype, une "Diaspora majuscule, un nom propre" (Dufoix, 2004), générant un certain nombre d'attributs, jugés par la plupart des membres de la catégorie dénotée, tel le maintien de liens réels ou symboliques institutionnalisés malgré la dispersion d'un peuple.

(5) Membre de la diaspora en question.

(6) Cet extrait est issu d'un entretien mené à Karikal, en Inde du Sud, en 2005. Mon interlocuteur vit en France, précisément à La Courneuve, dans la banlieue nord de Paris. II vient rendre visite à sa famille.

(7) Mon interlocuteur habite Paris, dans le XIXe arrondissement.

(8) Avec l'ouverture de la campagne "Incredible India" et les jeux du Common-wealth, le gouvernement indien table sur un objectif de 10 millions de touristes pour 2010.

(9) Le High Level Committee on the Indian Diaspora (HLCD) a été créé le 18 août 2000, dans le but de dresser un état des lieux historique et actuel des communautés indiennes d'outremer, de recenser les problèmes et les attentes de la diaspora vis-à-vis de l'Inde et de proposer au gouvernement de nouvelles orientations.

(10) Véritable "fête de la diaspora", cet événement est célébré du 7 au 9 janvier de chaque année (le 9 janvier correspondant de manière symbolique à la date du retour de Gandhi d'Afrique du Sud en Inde). À l'occasion de la cérémonie, chaque début d'année, une dizaine de personnalités sont sélectionnées par un jury et se voient remettre par le Président de la République le Pravasi Bharat Samman [prix des émigrés d'honneur] pour leur dévouement à la cause de la diaspora et de l'Inde.

(I I) En France, pour des raisons pragmatiques, la fête est célébrée le dimanche le plus proche de la date du calendrier hindou, puisqu'elle nécessite de fermer momentanément à la circulation le quartier où se déroule la procession. La fête de Ganesh est une fête indienne célébrée dans toute l'Inde. Elle est antérieure au miracle.

(12) Cette prise de l'espace diasporique par le tourisme s'effectue par le biais des technologies de l'information et de la communication.

(13) Ce circuit a été mis au point par le directeur de l'agence qui est francophone mais qui n'est pas un français d'origine pondichérienne. Ce circuit s'adresse en priorité aux membres du réseau de clientèle de l'agence Souvenir Travels composé d'une vingtaine de personnes. (14) Classement effectué d'après une enquête réalisée au sein de l'ensemble des agences de voyages de Pondichéry en juin 2010.

(I5) [http://www.paris.fr/portail/loisirs/Portal.lut? page_id=967 I \&document_type_id=5\& document_id=32 1 46\&portlet_id=23830)], consulté le 21 octobre 2010.

(16) La plupart des circuits et séjours proposés par ces agences de voyages sont à destination de l'Inde et de Sri Lanka. Elles proposent également un service d'achat de billets d'avion, à destination de l'ensemble des pays sud-asiatiques,

(17) "The literature on diaspora and hybridity has on the whole neglected tourism, perhaps because tourist visits are thought to be temporary and superficial" [La littérature sur les diasporas et l'hybridité a dans son ensemble négligé le tourisme, peut-être parce que les pratiques touristiques sont considérées comme temporaires et superficielles.] (Bruner, 1996, p. 290). 


\section{RÉFÉRENCES BIBLIOGRAPHIQUES}

Benedict ANDERSON, L'Imaginaire national : réflexions sur l'origine et l'essor du nationalisme, La Découverte, 2002.

Paul BASU, "Route metaphors of roots-tourism in the Scottish diaspora", dans Simon ColEMAN et John EADE (dir), Reframing Pilgrimage: Cultures in Motion, Routledge, 2004

Edward BRUNER, "Tourism in Ghana. The Representation of Slavery and the Return of the Black Diaspora", American Anthropologist, vol. 2, 1996.

Georgia CerIANI-SEBREGONDI, "La circulation migratoire aujourd'hui. Émergence d'un capital mobilitaire et d'une culture de la mobilité. Le cas des migrants marocains en Europe du Sud", Cédric AudEberT et Emmanuel MA MUNG (dir.), Les nouveaux territoires migratoires : entre logiques globales et dynamiques locales, Université de Deusto, 2006. JAMES CLIFFORD, Routes: travel and translation in the late twentieth century, Harvard University Press, 1997.

Robin COHEN, Global diasporas. An introduction, Routledge, 1997.

Stéphane Dufolx, "Notion, concept ou slogan : qu'y a-t-il sous le terme de "diaspora" ?", dans Lisa ANTEBY-YeMINI, William

Berthomière et Gabriel Sheffer (dir.), Les Diasporas. 2000 ans d'histoire, Presses universitaires de Rennes, 2005.

Stéphane Duforx, "Chronique bibliographique : l'objet diaspora en question", Cultures et conflits, 33-34, 2004.

Stéphane Dufolx, Les Diasporas, coll. Que sais-je? Puf, 2003. Stéphane DuFoIX et Foucher VALÉRIE, "Les Petites Italies (et les autres...). Éléments de réflexion sur la notion d'ethnoterritoire", dans Marie-Claude Blanc-ChalÉARD (dir), Les Petites Italies dans le Monde, Presses universitaires de Rennes, 2007.

MiCHEL Foucault, "Dits et écrits. Des espaces autres (conférence au Cercle d'études architecturales, 14 mars 1967)" Architecture,

Mouvement, Continuité, 5, 1984,

Anthony Goreau-PONCEAUD, La Diaspora tamoule : trajectoires spatio-temporelles et inscriptions territoriales en Île-de-France, thèse de géographie, 2008.

Maurice HaLBWACHS, Les cadres sociaux de la mémoire,

Albin Michel, 1994.

Rémy KNAFOU et Mathis STOCK, "Tourisme", dans Jacques LÉVY et

Michel LusSAULt, Dictionnaire de la géographie et de l'espace des sociétés, Belin, 2003.

Martine HovanESSIAN, "La notion de diaspora : la question de la temporalité", dans Arméniens et Grecs en diaspora : approches comparatives, Actes du colloque européen et international organisé à l'École française d'Athènes (4-7 octobre 200 I), 2007.

Martine HovANESSIAN, "La notion de diaspora : les évolutions d'une conscience de la dispersion à travers l'exemple arménien", dans Lisa ANTEBy-Yemini, William BerThomière et Sheffer GABriel, Les Diasporas. 2000 ans d'histoire, Presses universitaires de Rennes, 2005 Emmanuel MA MuNG, "Non lieu et utopie : la diaspora chinoise et le territoire", dans Michel BrUNEAU, Diasporas, GIP-Reclus, 1995.

Emmanuel MA MuNG, "La dispersion comme ressource", Culture et conflits, $n^{\circ} 33-34,1999$.

George MARCus, Ethnography through thick and thin, Princeton University Press, 1998.

Abraham Moles, "Nissonologie ou science des Îles", L'espace géographique, $n^{\circ} 4,1982$.

Lucie K. MORISSET et Luc NOPPEN, "De la production des monuments. Paradigmes et processus de la reconnaissance",

Khadiyatoulah FALL, Jocelyn LÉTOURNEAU et Laurier TURGEON (dir), Les Espaces de l'identité, Presses de l'université de Laval, 1997.

Anne RAULIN, L'Ethnique est quotidien. Diasporas, marchés et cultures métropolitaines, coll. Connaissance des hommes, L'Harmattan, 2000. Mathis STOCK, "L'hypothèse de l'habiter poly-topique : pratiquer les lieux géographiques dans les sociétés à individus mobiles",

EspacesTemps.net, Textuel, 2006

[http://espacestemps.net/document I 853.html].

Mathis Sтоск, "Les sociétés à individus mobiles : vers un nouveau mode d'habiter?", EspacesTemps.net, Textuel, 2005

[http://espacestemps.net/document I 353.html].

Mathis Stock (dir.), Le Tourisme. Acteurs, lieux et enjeux. Belin sup, Belin, 2003.

Mathis Stock, Philippe VıOLIER et Philippe Duhamel, "Variations d'altérité et "fin" du tourisme", Colloque Fins et confins du tourisme, Grenoble, 26-27 mai 2009.

JEAN-DIDIER URBAIN, "Le résident secondaire, un touriste à part ?", Ethnologie française, 2, 2002.

JOHN URRY, Sociologie des mobilités : une nouvelle frontière pour la sociologie, Colin, 2005.

DENIS VIDAL, "Empirisme et croyance dans l'hindouisme contemporain : quand les dieux boivent du lait", Annales HSS, vol. 52, 4, 1997. 